Mitteilungen der Österreichischen Geographischen Gesellschaft, 160. Jg., S. 406-424

(Annals of the Austrian Geographical Society, Vol. 160, pp. 406-424)

Wien (Vienna) 2018, https://doi.org/10.1553/moegg160s406

\title{
BUCHBESPRECHUNGEN BOOK REVIEWS
}

BäTzıng Werner (2018): Die Alpen. Das Verschwinden einer Kulturlandschaft. Darmstadt: Theiss / Wissenschaftliche Buchgesellschaft. 1. Aufl., 216 S., 228 meist farbige Fotos. ISBN: 978-3-8062-4779-5.

Mit der vorliegenden Veröffentlichung legt der Autor, der zahlreiche häufig gelesene Bücher über die Alpen verfasst hat, einen neuen Bildband vor, der sich hinsichtlich der Gestaltung von vergleichbaren Veröffentlichungen deutlich unterscheidet. Während diese die Sehnsüchte der Städter bedienen, dokumentiert Werner BäTZING die Auswirkungen des modernen sozioökonomischen Wandels anhand von eindrucksvollen Fotos, von denen er die meisten in den vergangenen 40 Jahren selbst aufgenommen hat. Bei älteren Vergleichsbildern griff er auf bekannte Fotoarchive zurück. Bei dieser Publikation handelt es sich um die Neuausgabe seines Bildbandes vom Jahr 2005, für welchen er drei Viertel der Fotos ausgetauscht bzw. aktualisiert und den Text neu geschrieben hat. Dabei erhoffte er sich, dass ,dieser Bildband nicht nur gefällt, sondern das Alpenerlebnis vieler Menschen vertieft und bereichert" (Vorwort).

Im Buch werden die aussagekräftigen Fotos nur kurz beschrieben und topographisch eingeordnet, jedoch nicht genauer interpretiert. Sie dienen als anschauliche Belege für die Texte des Buches, das in fünf Kapitel gegliedert ist. Das erste Kapitel behandelt die Frage: Was sind die Alpen? Dabei spannt er den Bogen von frühen Vorstellungen über die „bedrohlichen“ und die „schrecklich-schönen“ Berge bis zur überschießenden Nutzung der „Alpen als Freizeitpark“. Das zweite, traditionell angelegte Kapitel behandelt die „Natur der Alpen“. Auf dieses folgen die drei Hauptkapitel, von denen das erste die „traditionellen Kulturlandschaften“, das zweite die „Modernisierung der Alpen“ und das dritte und letzte „die aktuelle Situation und die Zukunft der Alpen“ behandelt. Die Texte fußen vorwiegend auf den Ergebnissen von BäTZINGs Primärforschungen, die sich auf die Westalpen und das Gasteiner Tal konzentrieren.

Die Gestaltung der alpinen Kulturlandschaften führt der Autor zu Recht auf die Aktivitäten der bäuerlichen Bevölkerung zurück, wobei er nur zwei Grundtypen unterscheidet. In den Altsiedelräumen herrscht der Ackerbau vor, der durch die Almwirtschaft in den Hochlagen ergänzt wird. Im Jungsiedelland dominiert hingegen die Tierhaltung und als Baumaterial spielt das Holz eine wichtige Rolle. Auf weitergehende Differenzierungen, wie die mit den agrarsozialen Verhältnissen zusammenhängende Unterscheidung zwischen den Alm- und Waldbauerngebieten in den Ostalpen, verzichtet er. Im Kapitel „Die Modernisierung der Alpen“ fasst er den modernen Strukturwandel zusammen, wobei er u. a. den Gang der Verkehrserschließung, den Verfall der Landwirtschaft und das Wachstum der Alpenstädte hervorhebt. Bezüglich des Tourismus stellt er fest, dass dieser kein Schlüsselgewerbe in den Alpen sei, in vielen Regionen spiele er vielmehr nur eine geringe Rolle. Das Schlusskapitel zur aktuellen Situation und den Zukunftsperspektiven behandelt aktuelle Fragen der alpinen Raumplanung.

Die vorliegende Monographie verdient besondere Beachtung. Die repräsentativ ausgewählten Fotos dokumentieren typische Beispiele des modernen Strukturwandels und erlauben es dem Be- 
trachter, sich eigenständig mit Problemen des Alpenraumes zu beschäftigen. Die Texte verdeutlichen die wissenschaftlichen Positionen des Autors und sollten die Fachvertreter anregen, sich mit seinen Aussagen kritisch auseinanderzusetzen, wobei manche Fachvertreter bei einzelnen Fragestellungen zu abweichenden Ergebnissen kommen dürften.

Hugo Penz (InNSBRuck)

Engartner Tim, Fridrich Christian, Graupe Silja, HedtKe Reinhold, TAfNer Georg (Hrsg.) (2018): Sozioökonomische Bildung und Wissenschaft. Entwicklungslinien und Perspektiven (= Sozioökonomische Bildung und Wissenschaft, Band 1). Wiesbaden, Springer VS. 1. Aufl., XVI und 245 S. ISBN 978-3-658-21217-9.

Mit dem vorliegenden Band wird eine neue Buchreihe gestartet, die es sich zur Aufgabe macht, auf der Grundlage eines gemeinsamen Verständnisses von sozioökonomischer Bildung unterschiedliche Ansätze in Lehre und Forschung vorzustellen. Die Herausgeberin und die Herausgeber charakterisieren diese Gemeinsamkeit als Verpflichtung zu den Prinzipien Interdisziplinarität, Pluralität, Multiparadigmatizität und Kontroversität sowie zur permanenten ethischen Reflexion bei der Behandlung ökonomischer Fragestellungen in ihren vielfältigen Zusammenhängen.

Die Reihe wird in Kooperation mit der Gesellschaft für ,Sozioökonomische Bildung und Wissenschaft" (GSÖBW) herausgegeben, die sich der Erneuerung der wirtschaftswissenschaftlichen Ausbildung und der Reform der ökonomischen Bildung verpflichtet fühlt. Sie vernetzt Vertreterinnen und Vertreter aus unterschiedlichen sozialwissenschaftlichen Disziplinen. In diesem Zusammenhang sei auch auf die österreichische „Fachgruppe für Geographische und Sozioökonomische Bildung“" (GESÖB) - einer Fachgruppe der Österreichischen Geographischen gesellschaft - verwiesen, die in enger fachlicher und auch personeller Kooperation zur GSÖBW steht. Einige ihrer Mitglieder gehören auch dem Herausgeberteam der neuen Buchreihe an.

Gemeinsame Basis aller Beiträge ist, „dass sie den ökonomischen Aspekt in einen sozialen, ethischen, historischen, kulturellen, politischen und/oder räumlichen Kontext einbetten“ (S. X). Die Autorinnen und Autoren führen den Methodenstreit in der Volkwirtschaftslehre (analytisch-fachlicher Zugang Kontra normativer Zugang im moralischen und ethischen Sinn sowie Relevanz von Deduktivem oder Induktivem) bewusst fort; eine abschließende Beurteilung steht nach wie vor aus. Letztlich wird immer wieder die wichtigste Frage gestellt, was nämlich unter den Phänomenen „Wirtschaft“ und „Wirtschaften“ zu verstehen ist.

Die Autorinnen und die Autoren liefern mit ihren Aufsätzen auch einen wichtigen Beitrag zum herrschenden Diskurs in wirtschaftswissenschaftlichen Lehrveranstaltungen an Universitäten, der sich zunehmend gegen die Dominanz der Neoklassik richtet, die alternative Denkansätze und Methoden bekämpft. Um mit Reinhold HeDTKe zu sprechen: „Die Freiheit des Denkens hängt auch davon ab, dass man zwischen unterschiedlichen Denkweisen wählen kann. Werden einem dagegen die Alternativen vorenthalten, weiß man nicht einmal, dass man frei wählen könnte, wenn man die unterschiedlichen Denkkonzepte kennen würde.“ (HedtKe 2008, S. 6).

In den Einzelbeiträgen werden auf der Basis des oben besprochenen Verständnisses von sozioökonomischer Bildung - Erschließung des Ökonomischen im sozialwissenschaftlichen, historischen, geographischen und kulturellen Kontext - spezielle Fragestellungen betrachtet:

Reinhold HEDTKE beschäftigt sich in seinem Aufsatz „Sozialwissenschaftlichkeit als sozioökonomisches Prinzip“ mit der Verankerung der sozioökonomischen Bildung in den Sozialwissenschaften und verwirft damit die Monodisziplinarität der orthodoxen ökonomischen Bildung. Er legt dar, warum das Prinzip der Sozialwissenschaftlichkeit durch die zugrundeliegenden Konzepte und 
Denkweisen aus bildungstheoretischer, fachdidaktischer und fachwissenschaftlicher Sicht zu einer echten sozioökonomischen und damit auch politischen Bildung führt.

Tim ENGARTNER skizziert in seinem Beitrag „Eckpfeiler sozioökonomische Bildung - oder: Zur Bedeutsamkeit der Kontextualisierung ökonomischer Frage- und Problemstellungen “, warum eine neoklassisch geprägte Wirtschaftsdidaktik, die ausschließlich auf diese Modell-und Disziplinorientierung ausgerichtet ist, große erkenntnistheoretische, bildungspolitische und fachdidaktische Mängel aufweist. Stellvertretend für viele andere Beispiele sei auf die Persistenz des „,homo oeconomicus" im Lehrkontext hingewiesen.

Silja Graupe versucht in ihrem Aufsatz „Die Welt mit anderen Augen sehen und meinen Weg finden “ „[...] erstmalig eine Existenzorientierung sozioökonomischer Bildung zu entwickeln“ (S. 53). Diese Existenzorientierung, also die Hinwendung zu Subjekt und Lebenswelt, könnte der Ausgangspunkt einer neuen sozioökonomischer Bildung werden und einen Gegenpol zur aktuellen ökonomischen Standardlehre bilden.

Christian FrIDRICH beschäftigt sich mit seinem Beitrag ,Sozioökonomische Bildung an allgemeinbildenden Schulen der Sekundarstufe I und II in Österreich - Entwicklungslinien, Umsetzungspraxis und Plädoyer für das Integrationsfach Geographie und Wirtschaftskunde" mit der Besonderheit dieser Fächerkombination für die sozioökonomische Bildung, bei der Wirtschaft als gesellschaftlich eingebettet und gestaltbar verstanden wird. Beispiele für die schulische Umsetzung, die im Rahmen einer größeren empirischen Studie erhoben wurden, zeigen die unterschiedliche Beliebtheit dieser sozioökonomischen Bildung bei Lehrenden und dokumentieren auch das Interesse der Schülerinnen und Schüler an sozioökonomischen Fragestellungen.

Georg TAFNER fordert in seinem Aufsatz „Ökonomische Bildung ist sozioökonomische Bildung. Grundlagen einer bildungswissenschaftlich fundierten Didaktik der Wirtschaftspädagogik" die Erweiterung des ökonomischen Parts um die soziale, politische und ethische Dimension. Durch das von ihm konzipierte „Rad der sozioökonomischen Bildung“ (S. 136), das - ausgehend vom Ich - die sechs Dimensionen einer reflexiven Wirtschaftspädagogik, die Mittel zur Erreichung des Ziels der jeweiligen Dimension und die Wirklichkeitsebenen von Effizienz, Verantwortung und Sinn darstellt, hat der Autor ein diskussionswürdiges Modell einer neuen Wirtschaftspädagogik entwickelt.

Thomas HöHNE betrachtet in seinem Beitrag „Ökonomisierung der Produktion von Schulbüchern, Bildungsmedien und Vermittlungswissen ", in welcher Weise die Ziele und Inhalte der (sozio-) ökonomischen Bildung durch den Wettbewerb am Schulbuchmarkt und die Bereitstellung von kostenlosen Materialien durch außerschulische Akteure beeinflusst werden. Er konstatiert eine Funktionalisierung der Bildung, in deren Mittelpunkt die Vorbereitung auf standardisierte Prüfungen und nicht die grundlegenden Prinzipien der sozioökonomischen Bildung in Bezug auf Lernprozesse, Gegenstandsbereiche und Wissenschaftsorientierung vermittelt werden.

Tonio Oeftering, Julia OpPermann und Andreas Fischer fokussieren ihren Beitrag ,, Gestaltbarkeit aller Lebensbereiche. Der Bildungswert der Lebensweltorientierung in der sozioökonomischen Bildung " auf das zentrale Prinzip der Lebensweltorientierung, das immer gefordert, aber selten näher betrachtet wird. Ihr Aufsatz ist auch ein Rückblick auf einen Workshop, der sich mit dem fachdidaktischen „Code“ der Lebenswelt- und Situationsorientierung im sozialwissenschaftlichen Unterricht beschäftigte. Das unterschiedliche Verständnis dieses „Codes“ bei Lehrenden bzw. die Herausforderungen bei der Umsetzung im Unterricht an der Schnittstelle zwischen Öffentlichkeit und Privatheit werden beispielhaft aufgezeigt.

Moritz Peter Hartmann argumentiert in seinem Aufsatz ,, Die Rekonstruktion, die Dekonstruktion und die Konstruktion der Wirtschaftsordnung als Kern der sozioökonomischen Bildung " für eine Didaktik, die Schülerinnen und Schüler dazu befähigt, ökonomisches Handeln vor dem Hintergrund der bestehenden Wirtschaftsordnung zu betrachten. Das bedeutet aber auch, aktuelle Macht-und Herrschaftsverhältnisse zu erkennen und sie als Konstrukte, die veränderbar sind, zu begreifen. 
Christoph Schank und Alexander LoRCH diskutieren im letzten Beitrag des Bandes „Der Nachhaltigkeitsbürger in der sozioökonomischen Bildung - Überlegungen zu einem wirtschaftsethisch fundierten sozioökonomischen Bildungsideal " die Frage, warum ausschließlich eine Erziehung zu freien Nachhaltigkeitsbürger/inne/n, die kritisch und emanzipiert und damit nachhaltig handeln können, eine erstrebenswerte sozioökonomische Bildung ist. Sie erweitern die bisherigen Vorstellungen von Wirtschaftsethik um einen kompetenzbasierten Beitrag zu einer nachhaltigen gesellschaftlichen Entwicklung.

Dieses Buch ermöglicht allen Lehrenden an Schulen und Universitäten, zu überprüfen, auf welchem theoretischen Fundament ihr sozioökonomischer Unterricht steht und welche wichtigen Prinzipien bisher kaum oder nicht berücksichtigt wurden. Die „Black Box“ Unterricht könnte durch diese Lektüre entscheidend ,erhellt“ werden.

\section{Zitierte Literatur:}

HedtKe R. (2008): Ökonomische Denkweisen. Eine Einführung. Multiperspektivität - Alternativen - Grundlagen. Schwalbach, Wochenschau-Verlag.

Maria Hofmann-Schneller (Wien)

FIELD Andy (2018): Discovering Statistics Using IBM SPSS Statistics. London - Thousand Oaks - New Delhi - Singapore, SAGE Publications. Fünfte Aufl., 1070 S., zahlr. Farbabb. und Tab. ISBN 978-1526419521.

Die Fülle der in FIELD's Handbuch bzw. Lehrbuch „Discovering Statistics Using IBM SPSS Statistics“ dargebotenen Informationen überwältigt. In 21 Kapiteln und auf über tausend Seiten findet man weit mehr, als man in Einführungslehrveranstaltungen zur Statistik zu hören bekommt. Beginnend bei den Grundbegriffen der Statistik und einer detaillierten Einführung in SPSS spannt das Buch einen weiten Bogen über nichtparametrische Modelle, die Korrelationsrechnung, die lineare Regression und den Vergleich zweier Mittelwerte hin zu generalisierten linearen Modellen (ANOVA, ANCOVA, „factorial designs“, Designs mit Messwiederholungen, „mixed designs“) zur multivariaten ANOVA, der exploratorischen Faktorenanalyse, der loglinearen Analyse, der logistischen Regression und der hierarchischen linearen Modellierung.

Mittlerweile in der 5. Auflage erschienen, besticht das Lehrbuch in mehreren Punkten, deren wichtigste zweifelsohne die nachstehend angeführten sind:

- Discovering Statistics Using IBM SPSS Statistics stellt eine gelungene Synthese zwischen Theorie und Praxis dar - so wird versucht, mit einem Minimalaufwand an mathematischer Theorie ein Maximum an Verständnis zu erzielen.

- FiELD's Lehrbuch bietet fast alles an statistischem Rüstzeug, das zwischen „first year undergraduate“ und ,postgraduate level“, um die Worte des Autors zu verwenden, im sozial- und wirtschaftswissenschaftlichen Bereich benötigt wird. Eine derartige Informationsfülle wäre jedoch nicht vermittelbar, wäre das Buch nicht dermaßen stringent verfasst: Kapitel, Unterkapitel sowie ergänzende Abschnitte, die Zusatzinformationen, Hinweise auf häufig gemachte Irrtümer, Zusammenfassungen, Selbsttests u.a.m. enthalten, bringen dem Leser die Inhalte auf eine Weise nahe, die auch für statistisch nicht so Versierte eine große Hilfe darstellt.

- Hilfreich für viele Anwender ist gewiss auch die Tatsache, dass im vorliegenden Buch weitgehend auf manuelle Berechnungen verzichtet, sondern gezeigt wird, wie sich (komplexe) statistische Aufgabenstellungen unter Zuhilfenahme von IBM SPSS lösen lassen. Beginnend mit einer 
grundlegenden Einführung in das Programmpaket, wird bei jedem der behandelten Verfahren gezeigt, wie es mittels IBM SPSS zu lösen ist. Dies umfasst zum einen eine „Step-by-step“-Anleitung für die Eingabe sowie zum anderen eine ausführliche Diskussion des erhaltenen Outputs, wobei zahlreiche Screenshots die Ausführungen ergänzen bzw. abrunden.

- Erwähnenswert ist zudem das Layout von Discovering Statistics Using IBM SPSS Statistics ein Layout, von dem man bei deutschsprachigen Statistikbüchern nur träumen kann. Eine Vielzahl mehrfarbiger Grafiken visualisiert Geschriebenes und unterstützt die Strukturierung der Inhalte, Charaktere (!) wie „Cramming Sam“, „, Jane Superbrain“ oder „Satan's Personal Statistcs Slave” kennzeichnen spezielle Inhalte wie Zusammenfassungen, ergänzende Informationen oder SPSS-Zusatzinformationen und fungieren somit als Überschriften von Unterabschnitten.

- Abschließend soll noch darauf hingewiesen werden, dass für den Fall, dass Discovering Statistics Using IBM SPSS Statistics in einer Lehrveranstaltung eingesetzt wird, sowohl für Studierende als auch für Lehrende umfangreiche Online-Ressourcen zur Verfügung stehen.

Resümierend lässt sich festhalten, dass es sich bei FIELD“s Lehr- bzw. Handbuch „Discovering Statistics Using IBM SPSS Statistics“ um ein ausgezeichnetes Kompendium zur Statistik handelt, welches in der Bibliothek keines quantitativ arbeitenden Geographen fehlen sollte.

Gert W. Wolf (Klagenfurt a. W.)

GruBER Elisabeth (2017): Im Ruhestand aufs Land? Ruhestandsmigration und deren Bedeutung für ländliche Räume in Österreich (= Rural Areas: Issues of Local and Regional Development / Ländliche Räume. Beiträge zur lokalen und regionalen Entwicklung, Band 2). Zürich, LIT-Verlag. 1. Aufl., 248 S., zahlreiche Farbabb. ISBN 978-3-643-50807-2.

Mit der Veröffentlichung ihrer Dissertation greift Elisabeth GRUBER das vor dem Hintergrund des demographischen Wandels zunehmend an Relevanz gewinnende Forschungsfeld der Ruhestandswanderung auf. Die Veröffentlichung, die im Jahr 2017 in der Reihe „Ländliche Räume. Beiträge zur lokalen und regionalen Entwicklung" im LIT-Verlag erschienen ist, beschäftigt sich mit dem Phänomen der Migration von Seniorinnen und Senioren in jene ländlichen Räume, die insgesamt häufig eine rückläufige Entwicklung der (jungen) Bevölkerung aufweisen. Die übergeordnete Zielsetzung des Buches ist eine genaue Untersuchung „der Bedeutung von Ruhestandswanderung für ländlich-periphere Gemeinden und Regionen in Österreich“ (S. 6). Neben der Auswertung altersspezifischer Migrationsdaten werden anhand zweier österreichischer Fallstudien - dem Südburgenland und dem Waldviertel - die Motive der Zuwandernden sowie die räumlichen und sozialen Auswirkungen des Zuzugs auf die Zielgemeinden thematisiert. Als Ruhestandsmigrantinnen und -migranten werden dabei sowohl neu zugezogene Personen, die bisher wenig Kontakt mit der Region hatten, als auch zurückkehrende Personen, bezeichnet.

Die Monographie gliedert sich in sechs Kapitel. Während in Kapitel 1 der Forschungsgegenstand überblicksmäßig präsentiert wird, werden in Kapitel 2 der aktuelle Forschungsstand sowie der theoretische Hintergrund erläutert. Kapitel 3 beschreibt das Forschungsdesign und die methodische Herangehensweise. In den beiden nachfolgenden Kapiteln 4 und 5 werden zunächst die empirischen Ergebnisse der österreichweiten quantitativen Analysen und nachfolgend die fallspezifischen qualitativen Untersuchungen vorgestellt. Das sechste Kapitel hält eine Synthese bereit, auf der aufbauend allgemeine Handlungsempfehlungen abgeleitet werden.

Die klar strukturierte Arbeit besticht durch den integrativen Ansatz aus quantitativen und qualitativen Methoden, der einen umfassenden Überblick über aktuelle Dynamiken und Auswirkungen von 
Altersmigration in Österreich ermöglicht. Nach einer knappen theoretischen Einführung zum aktuellen Forschungsstand und zu den relevanten Konzepten aus der Migrationsforschung (u.a. „Life Course Migration“, „Amenity Migration“) fokussiert der Beitrag auf die umfangreiche Aufbereitung der empirischen Ergebnisse. Auf Basis von statistischen Daten werden räumliche Entwicklungen und Konzentrationen von altersspezifischer Mobilität anhand von Grafiken und kartographischen Darstellungen übersichtlich dargestellt und diskutiert. Insbesondere die daran anknüpfende qualitative Auseinandersetzung mit den nachvollziehbar ausgewählten Fallregionen stellt eine große Stärke der Studie dar. Anhand zahlreicher Interviewzitate werden nicht nur anschauliche Profile der Zugewanderten erstellt und ihre Wanderungsmotive analysiert, sondern auch die bestehenden Herausforderungen und angedachten Strategien aus Sicht der Zielgemeinden erörtert. An manchen Stellen wäre jedoch eine vertiefende Einbettung der empirischen Ergebnisse in den theoretischen Kontext wünschenswert gewesen, um die Anschlussfähigkeit der vorgelegten Ergebnisse im Migrationsdiskurs zu erhöhen. Ferner wäre aufgrund der breiten Basis der Resultate auch eine Konkretisierung der abgeleiteten Handlungsempfehlungen denkbar gewesen, die eine weiterführende Diskussion in der Praxis anregen könnte.

Zusammenfassend stellt die Dissertation von Elisabeth GRUBER einen sehr lesenswerten und interessanten Beitrag aus Österreich sowohl zur wissenschaftlichen als auch zur anwendungsorientierten Debatte der Ruhestandsmigration dar. Eine ausdrückliche Empfehlung richtet sich daher nicht nur an Geographinnen und Geographen in der Wissenschaft, sondern auch an Praktikerinnen und Praktiker aus Politik und Regionalentwicklung, die sich mit alternativen Entwicklungsstrategien für ländliche Räume befassen.

Stefanie DöRINGER (Wien)

Heintel Martin, Musil Robert, Weixlbaumer Norbert (Hrsg.) (2018): Grenzen. Theoretische, konzeptionelle und praxisbezogene Fragestellungen zu Grenzen und deren Überschreitungen (Reihe: RaumFragen: Stadt - Region - Landschaft). Wiesbaden, Springer VS. 1. Aufl., VII und 463 S., 64 Abb., 7 Abb. in Farbe. ISBN 978-3-658-18432-2.

Staatliche Grenzen gewinnen zuletzt wieder politisch an Bedeutung und damit ist dieser umfangreiche Sammelband höchst aktuell und willkommen. Er umfasst neben einer Einführung in das Buch und die Thematik drei Teile: Teil I mit den theoretischen und konzeptionellen Zugängen zur Grenze, Teil II über Grenzen und Planungspraxis und Teil III über Grenzen und Handlungspraxis. Jeder Teil umfasst zirka 150 Seiten und wäre damit schon fast ein eigenständiges Buch zur Thematik. Dies zeigt den Umfang und die Vielfalt der Beiträge und angesprochenen Themen, von denen nur wenige, subjektiv ausgewählte, Artikel hier genannt werden können.

So wäre beispielsweise aus Teil I das Kapitel von Heinz NISSEL über Grenzen als Konstante der Politischen Geographie und Geopolitik sehr gut in jeder Vorlesung zur Politischen Geographie einsetzbar. Dies gilt auch für den Beitrag von Ulli VILSMAIER, der als Grundlage für die Reflexion und theoretische Einordnung eigener Grenzforschung dienen kann. In Teil II hinterfragt Birte NiENABER Grenzen als überwindbares Phänomen in der Raumordnung und stellt insbesondere die Instrumente der EU zur grenzüberschreitenden Zusammenarbeit in der Planung vor, während Axel Priebs die grenzüberschreitende Zusammenarbeit über Verwaltungsgrenzen aus der Sicht der Planungs- und Verwaltungspraxis thematisiert. In den folgenden Artikeln werden Planungsbeispiele aus der „Regio Basiliensis“, der Bodenseeregion, der Kernregion Salzburg, der Suburbanisierungsregion von Bratislava und der Euroregion POMERANIA vorgestellt. Teil III wird mit einem Beitrag über den Zusammenhang von Grenze und Migration von Heinz Fassmann eingeleitet und mit Beispielen von der polnisch-belarussischen und der polnisch-ukrainischen, aber auch der belgisch-niederländischen-deutschen Grenze fortgesetzt. Mit einem Beitrag über die Grenze zwischen San Diego und 
Tijuana kommt auch die derzeit sehr prominente und vieldiskutierte Südgrenze der USA in den Fokus. Schließlich wird ein hochschuldidaktisches Projekt von Martin HeINTEL, Milan Jě̌ÁBEK und Norbert WeIXLBAUMER im südmährisch-niederösterreichischen Grenzraum vorgestellt und reflektiert.

Der Band wird mit einem besonderen „bijou“ abgeschlossen, nämlich zwölf Skizzen zu Kontinuität und Wandel niederösterreichischer Grenzlandschaften, die letztlich zu zwei Thesen führen: „These 1: Grenzen sind gesellschaftliche Konstrukte, theoretisch und forschungspraktisch interessant ist jedoch, was die Konstruktion ausmacht [...]. These 2: Grenzen sind nichts Natürliches. Natürliches spielt jedoch für Grenzziehungen eine nicht zu vernachlässigende Rolle.“ (S. 458f)

Das Besondere des Bandes sind die umfassende theoretische Einführung sowie die konzeptionellen Reflexionen in Teil I. Diese spiegeln sich in der theoretischen Fundierung der empirischen Beiträge und dem hohen Reflexionsgrad aller Kapitel. Gleichzeitig macht die gewählte Perspektive der Grenzforschung auf Planungsfragen und Handlungsfelder dieses Buch für die Praxis und Hochschullehre relevant und wertvoll. Den Herausgebern ist damit ein wichtiger Beitrag zur theoretischen, konzeptionellen und praxisbezogenen Grenzforschung gelungen.

Doris WastL-WaLter (Bern)

Husa Karl, Trupp Alexander, Wohlschlägl Helmut (Hrsg.) (2014): Southeast Asian Mobility Transitions: Issues and Trends in Migration and Tourism (= Abhandlungen zur Geographie und Regionalforschung, Band 19). Wien, Institut für Geographie und Regionalforschung der Universität Wien. 1. Aufl., 452 S., zahlreiche z. T. farbige Abb. und Tab. ISBN: 978-3900830-84-7.

Seit Beginn der 1980er Jahre hat sich mit der „Südostasien Forschungsgruppe“ am Institut für Geographie und Regionalforschung der Universität Wien ein Kompetenzzentrum geographischer Südostasienforschung entwickelt, für das es, betrachtet man Quantität und Qualität der vorgelegten Forschungsergebnisse, im deutschsprachigen Raum nur wenig Vergleichbares gibt. Auch der vorliegende Sammelband, der sich anhand ausgewählter Beispiele mit dem Wandel räumlicher Mobilitätsmuster in Südostasien befasst, zeugt von dieser bemerkenswerten Produktivität. Zahlreiche aus der Region stammende Autorinnen und Autoren sind ein Beleg für die fruchtbaren wissenschaftlichen Kontakte und Kooperationen mit Forschungseinrichtungen in Südostasien, die sich im Lauf der Jahre entwickelt haben. Die Herausgeber selbst sind neben der gemeinsamen Einführung auch als Autoren oder Mitverfasser mehrerer Beiträge des Bandes vertreten.

Die Grobgliederung des Bandes folgt der üblichen Zuordnung verschiedener Mobilitätsformen zu den Kategorien „Migration“ und „Tourismus“. Allerdings betonen die Herausgeber in ihrer Einführung sehr zu Recht, dass klare Unterscheidungen zwischen verschiedenen Mobilitätsformen zunehmend fragwürdig geworden sind, vielfach ergeben sich im Zeitverlauf auch fließende Übergänge. Dazu beigetragen hat sicher die Herausbildung neuer Akteurstypen wie etwa Langzeittouristen oder Ruhestandsmigranten. Aber auch zwischen freiwilliger und erzwungener Migration, zwischen Migration aus wirtschaftlichen oder aus anderen Gründen lassen sich oft keine eindeutigen Unterscheidungen mehr treffen. Auch bei der „klassischen“ Land-Stadt-Migration sind zunehmend komplexere Muster erkennbar, wie Ryoji SoDA am Beispiel der Iban in Sarawak/Malaysia zeigt.

In dem vorliegenden Band gehen mehrere Beiträge solchen Fragen nach, etwa anhand der Ruhestands- oder Heiratsmigration. Petra DANNECKer und Wolfram SchafFar machen am Beispiel burmesischer Migranten in der grenznahen thailändischen Stadt Mae Sot deutlich, wie wenig die Zuordnung zu den Kategorien „Arbeitsmigrant“ oder „Flüchtling“ auf objektivierbaren Kriterien 
beruht. Sie ist vielmehr immer Ausdruck einer zwischen zahlreichen Akteuren ausgehandelten, immer umstrittenen und deswegen auch nie endgültig festgeschriebenen sozialen Konstruktion. Damit wird eine Frage aufgegriffen, die weit über das behandelte Beispiel hinaus von erheblicher Bedeutung ist, wie sich an den kontroversen Debatten über Flucht und Migration ablesen lässt, die derzeit in Europa geführt werden.

Räumlich bilden Fallstudien aus Thailand einen Schwerpunkt des Bandes, was dem über Jahre gewachsenen intensiven Kontakten der „Forschungsgruppe“ geschuldet sein dürfte. Der Wandel räumlicher Mobilitätsmuster ist allerdings ein Südostasien insgesamt betreffendes Phänomen, wie weitere Beiträge mit Beispielen aus den Philippinen, Malaysia, Laos und Vietnam zeigen. Es geht dabei um neue Trends im Tourismus (u. a. neue Formen des kulturell motivierten Tourismus), nicht zuletzt aber auch um den Wandel der Arbeitsmigration: Neben der anhaltenden Land-Stadt-Migration ist Südostasien heute nicht mehr nur Quellgebiet weltweiter Arbeitsmigration, sondern selbst auch zum Zielgebiet grenzüberschreitender Arbeitskräftewanderungen geworden, wie Karl HusA und Helmut WoHLSCHLÄGL in einem zusammenfassenden Übersichtsartikel über „Global Markets - Local Consequences: The Migration of Labour in Southeast Asia Since the Mid-Nineteenth Century" und Kwanchit SASIwONGSAROJ am Beispiel irregulärer kambodschanischer, laotischer und burmesischer Arbeitsmigranten in Thailand zeigen.

Der Sammelband belegt eindrucksvoll, dass es gute Gründe gibt, in der Forschung von Mobilität auf allen räumlichen Maßstabsebenen und in all ihren Facetten als Normalfall und nicht als Ausnahme räumlichen Verhaltens auszugehen. Als Desiderat bisheriger Forschungen sei auf die zunehmende Bedeutung erzwungener Mobilität verwiesen, deren Gründe neben Krieg und Verfolgung auch in Umweltkatastrophen und wirtschaftliche Notlagen zu suchen sind. Der vorliegende Band vermittelt wichtige Einsichten in den Wandel räumlicher Mobilitätsmuster in Südostasien. Er ist darüber hinaus aber auch ein ausgesprochen begrüßenswerter Beitrag zur interdisziplinären Mobilitätsforschung, denn die behandelten Themen und aufgeworfenen Fragen sind über die Region hinaus von großer Relevanz.

Helmut SCHNEIDER (Duisburg-Essen)

KaHL Thede (2018): Natur und Mensch im Donaudelta (= Reihe Forum: Rumänien, Band 36). Berlin, Frank \& Timme-Verlag für wissenschaftliche Literatur. 1. Aufl., 244 S., 272 Fotos, 8 Karten. ISBN: 978-3-7329-0438-9.

Der Balkanologe Thede KAHL, der bereits mehrere grundlegende Werke zu Südosteuropa und Rumänien publiziert hat (darunter gemeinsam mit Michael Metzeltin und Mihai-Răzvan UnGUREANU 2006 das Buch „Rumänien“ im LIT-Verlag - im selben Buch auch einen Beitrag gemeinsam mit Josef SallanZ zur Dobrudscha, S. 857-879 - und kürzlich das „Handbuch Balkan“ mit Uwe HINRichs und Petra Himstedt-VAId bei Harrassowitz) legt nun eine Landeskunde des Donaudeltas vor.

Das in Rumänien und der Ukraine gelegene Donaudelta ist mit seiner Gesamtfläche von 5.640 Quadratkilometern Europas größtes Feuchtgebiet. Wenn es auch für diese einzigartige, ständigen Veränderungen unterworfene Landschaft eine wachsende Zahl an Publikationen gibt, so lag bisher der Schwerpunkt auf Bildbänden oder wissenschaftlichen Aufsätzen zu sehr spezifischen Themen, während eine einführende Landeskunde fehlte. KAHL gelingt es nun, diese Lücke zu schließen, indem er die erste länderkundliche Übersicht für diesen Lebensraum vorlegt. Man merkt dem Buch an, dass es mit viele Liebe und Verständnis für die Landschaft und seine Bewohner geschrieben wurde, wobei die kritische Distanz nicht fehlt. Das Buch steht somit zwischen einer wissenschaftlichen Annäherung und dem Versuch, Raum und Mensch über die wissenschaftliche Sicht hinaus verständlicher zu machen. 
Dem länderkundlichen Schema folgend geht KaHL auf Lage und Gestalt, Klima und Vegetation, Geschichte und Architektur, Flora und Fauna sowie die ethnische und wirtschaftliche Struktur der Bevölkerung ein. In allen Kapiteln zeigt sich, dass dieser amphibische Lebensraum stärker von der Donau als von den Menschen bestimmt wird. Das Buch erhebt den Anspruch, alle einheimischen Vögel, die regelmäßig im Delta vorkommen, und einen Großteil der Pflanzenarten zu verzeichnen.

Die in der Natur demonstrierte Vielfalt spiegelt sich in der ethnischen und religiösen Struktur wider. Der Autor konzentriert sich auf Ethnien, die historisch gesehen am längsten für die Region von Bedeutung sind. Dazu gehören die ab dem 17. Jahrhundert aus Glaubensgründen eingewanderten altgläubigen Lipowaner. Sie sind heute eine russischsprachige Minderheit, während die erst später ins Delta zugewanderten Rumänen inzwischen die Mehrheit bilden. Doch ganz gleich, ob Lipowaner, Rumänen oder die ursprünglich aus der Ukraine stammenden Chacholen (Nachfolger der Kosaken), viele von ihnen halten trotz der inzwischen internationalen wirtschaftlichen Bedeutung des Flusses an Lebensformen fest, die sich in den zurückliegenden 400 Jahren kaum verändert haben.

Neben dem Kapitel zur wirtschaftlichen Situation widmet der Autor ein weiteres Kapitel der Bedrohung des empfindlichen Biosystems sowie bereits ergriffenen und geplanten Schutzmaßnahmen. Ein Beispiel sind die Schäden, die durch das Trockenlegen großer Flächen des Deltas zur Gewinnung von Ackerland entstanden sind und die begonnene Renaturierung.

Durch seine hervorragenden, teils vergleichenden Fotos, das fantastische Kartenmaterial und ein eindrucksvolles Verzeichnis wissenschaftlicher Tier- und Pflanzennamen ist das Buch eine wahre Fundgrube für alle, die sich für Land und Leute dieser Region interessieren. Hierzu trägt auch das Literaturverzeichnis bei, dass die wichtigsten Quellen in allen vorstellbaren Sprachen zusammenträgt.

Zusammenfassend gelingt dem Autor, eine gut lesbare und übersichtliche wissenschaftliche Gesamtdarstellung in der Tradition des ländeskundlichen Schemas zu verfassen und dabei physiound anthropogeographische Aspekte gleichermaßen zu berücksichtigen. Eine Vielzahl von Aspekten wird in maximaler Breite vorgestellt. Aufgrund des Anspruchs, eine übersichtliche Landeskunde zu verfassen, leuchtet es ein, dass Kahl dabei auch Auslassungen nicht umgehen konnte, und der Leser beispielsweise nichts über lokale Einzelthemen erfährt.

Anastasia Plakasoviti (Jena / Jekaterinburg [Екатеринбу́рг])

Komlosy Andrea (2018): Grenzen. Räumliche und soziale Trennlinien im Zeitverlauf. Wien, Verlag Promedia. 1. Aufl., 247 S., 1 Abb. ISBN 978-3-85371-434-8.

Grenzen und Territorialität sind dem menschlichen Wesen eigen. Ohne Grenzen könnten wir keine Individuen sein, keine eigene Persönlichkeit entfalten. Das Streben nach einem eigenen Territorium, das einem gehört oder auf das man zumindest mehr Rechte hat als andere, beginnt beim Wunsch nach einem eigenen Zimmer in der Wohnung oder zumindest nach einem eigenen Schrank oder Schreibtisch, in denen man seine Habseligkeiten verwahren kann und zu denen jemand anderer nicht so ohne weiteres Zugriff hat. Der Wunsch nach Grenzenlosigkeit und einer Welt ohne Grenzen, die Neigung, Grenzen zu überschreiten und nicht zu beachten sind Mächtigen eigen oder Menschen, die sich im Besitz der Wahrheit wähnen, diese möglichst unbegrenzt verbreiten wollen oder sich moralisch überlegen fühlen. Schwache und von sich weniger Überzeugte schätzen hingegen den Schutz von Grenzen - ob in persönlichen Beziehungen, im Arbeitsleben oder als Teil einer Gemeinschaft. Das ist - um es auf ein aktuelles politisches Thema herunterzubrechen - auch das Hauptproblem der Sozialdemokratie in der Migrationsfrage. Der programmatische Internationalismus, einem Wahrheitsanspruch und moralischen Überlegenheitsgefühl entsprungen („Mit uns zieht die neue Zeit!"), und die gelegentlichen Versuche, entsprechend zu handeln, kollidieren mit dem sozialen 
Schutzbedürfnis der traditionellen politischen Klientel. Ein bezeichnender historischer Fall ist das römische Weltreich, das sich in seiner expansiven Phase als grenzenlos sah und sich erst in seiner Arrondierungs- und Konsolidierungsphase ab dem 2. Jahrhundert selbst Grenzen setzte.

Die geographische Relevanz von Grenzen ist evident: Sie sind wesenhaft mit Territorialität, räumlicher Bindung und raumbezogener Identität verbunden; mit Grenzen strukturieren wir den geographischen Raum, teilen ihn in geographische Objekte aller Art. Es gibt jedoch auch Grenzen, die nicht raumbezogen sind, z.B. Grenzen im mitmenschlichen Kontakt, in der Frage, was wir den anderen noch zumuten können und was nicht mehr. Man könnte dies auch durch das Beispiel der Sprache ergänzen, die zugleich ein- und ausschließt, Grenzen zwischen Menschen zieht - auf jeder ihrer Ebenen, auch durch Dialekt oder Intonation. Das in den verschiedenen Teilen des deutschen Sprachraums unterschiedlich intonierte Standarddeutsch hat genau diese Wirkung. Aber da sind wir schon wieder im Bereich des Räumlichen. „Ohne Grenzen kann nichts bewahrt und nichts überschritten werden" fasst Andrea KomLosy prägnant zusammen (S. 9).

Die Autorin, Professorin am Institut für Wirtschafts- und Sozialgeschichte der Universität Wien und bereits durch ihr Buch über Arbeit in globalhistorischer Perspektive hervorgetreten, möchte den Wunsch- und Feindbildern von Grenze deren geschichtliche Entwicklung und mannigfaltige Ausprägung entgegenstellen, sowohl ihr Herrschafts- als auch ihr Schutz- und Befreiungspotenzial. Sie orientiert die geschichtlichen Rückgriffe aber immer an aktuellen Fragestellungen. Geschichte wird also, wohl ein wesentliches Desiderat an die Geschichtsforschung, für die Gegenwart nutzbar gemacht.

Ein erstes Kapitel widmet sich den Begriffen. Das deutsche Wort Grenze, abgeleitet aus dem slawischen granica, steht für einen Begriff, der sich von Mark, Grenzsaum, Grenzgebiet zu Grenzlinie wandelte. Der alte Begriff klingt noch in der habsburgischen Militärgrenze gegen das Osmanische Reich nach, die ja ein oft breiter Grenzstreifen mit einer eigenen Verwaltung war, im slawischen Wort und Begriff krajina (Vojna krajina, Srpska krajina, Ukraine) und, das könnte man ergänzen, auch im rumänischen mărginimea (Mărginimea Sibiului). Grenze und Territorialität bilden ein voneinander abhängiges Begriffspaar. Letztlich bestimmt die Art menschlicher Territorialität den Charakter der Grenze.

Es folgt eine Chronologie der Territorialität mit deren grundlegenden historischen Modellen der überregional agierenden Stammesgesellschaften, des Stadtstaats und des Imperiums, wobei das noch nicht unbedingt die zeitliche Abfolge darstellt. In zeitlicher Abfolge wird aber doch die globale, insbesondere die europäische Herrschaftsgeschichte im Zeitraffer entwickelt, wobei die politisch-gesellschaftlichen Unterschiede zwischen dem lateinisch-römischen Westen und dem byzantinisch geprägten Osten Europas deutlich werden. Die Autorin unterstreicht auch die Eigenart des westeuropäischen Herrschaftssystems mit abgemilderter Zentralmacht und viel Subsidiarität, das sich im Laufe des Mittelalters herausbildete und später zum globalen Modell wurde, an dem sich möglichst alle (grenzenlos!) orientieren sollten, weil es ja (selbstredend!) allen anderen überlegen ist. Sie macht auch bewusst, dass sich der fest umgrenzte Flächenstaat heutigen Zuschnitts erst nach dem völlig durchmischten Territorialsystem des Mittelalters in der frühen Neuzeit zu entwickeln begann. Erst da wurde der Staat auch zu einem Wirtschaftsraum. Herrschaft äußerte sich aber nicht nur in Kontrolle über ein Territorium, sondern auch über externe Netzwerkknoten, Finanz- und Informationsflüsse. Das 19. Jahrhundert fügte dem noch die Ausgestaltung der Bürgerrechte hinzu, verband also Staatsvolk und Staatsterritorium. Damit - und durch eine gemeinsame Sprache - entstanden auch Staats- und Nationalbewusstsein. Der mit den 1970er Jahren einsetzende Globalisierungsprozess reduzierte wiederum die Bedeutung von Staaten und Staatsgrenzen.

Das Kapitel befasst sich aber nicht nur mit Territorialität und Grenzen im engeren Sinn, sondern bietet auch einen interessanten Blick auf die Gesellschafts- und Wirtschaftsentwicklung. Besonders treffend werden die steuernden Faktoren sowie die Auswirkungen der modernen Globalisierung auf 
globale und innerstaatliche sozio-ökonomische räumliche Disparitäten erklärt. Die Beschränkung staatlicher Souveränität und der Bedeutungsverlust staatlicher Grenzen werden offensichtlich, wenn man mit der Autorin bedenkt, dass so gut wie alle internationalen Organisationen, vereint mit den wirtschaftlich starken Staaten der Welt (derzeit vielleicht mit Ausnahme der USA) und den global agierenden Unternehmen die Öffnung der Märkte und die Freizügigkeit von Kapital, Gütern, Dienstleistungen und Arbeitskraft betreiben. Sozial und wirtschaftlich Schwache (Menschen und Staaten) können dem kaum noch etwas entgegensetzen. Vielleicht wird dadurch so manche, oft irrational anmutende Gegenbewegung besser verständlich.

Die Autorin sieht eine Epoche ,nach dem Staat“ anbrechen, in der Territorialität neu definiert werden muss. So wie einst der Staat als Rahmen für das Handeln von Wirtschaftsunternehmen geschaffen wurde, so würden sich heute seine Vertreter den Interessen der globalen Wirtschaftszentralen fügen, wenn der Staat Standort solcher ist. Der globale Wettbewerb um Investitionen führe durch ungleichmäßige Begünstigung auch zu einer „durchlöcherten“, dem Mittelalter nicht unähnlichen Territorialität. Auch weitere historische Analogien machen den auf Gegenwart und Zukunft bezogenen Schluss dieses Kapitels besonders interessant.

Eine Typologie der Grenzen unterscheidet zunächst zwischen sozialen (vertikalen) und räumlichen (horizontalen) Grenzen, die aber oft zusammenfallen - wie bei der sozialen Segregation in Städten. Sodann beleuchtet die Autorin verschiedene Arten politischer Grenzen von jener der Gemeinde bis zu der der Europäischen Union (EU). Wir erfahren, dass frühe Grenzen oft nicht linear, sondern nur punktuell definiert waren (an strategischen Stellen wie Pässen und Furten) und dass im Mittelalter Herrschaft über Personenverbände, nicht über Territorien ausgeübt wurde, Territorialität und damit Grenzen sich daher überlappten.

Als ein weiterer Typ werden Militärgrenzen genannt wie der römische Limes, die Marken des Heiligen Römischen Reiches oder die habsburgische Militärgrenze gegen das Osmanische Reich, aber auch die frontera und frontier der europäischen Zuwanderer nach Süd- bzw. Nordamerika.

Ihre Eigenart haben der Autorin zufolge auch Kolonialgrenzen, die ohne Rücksicht auf geographische, wirtschaftliche oder kulturelle Gegebenheiten gezogen wurden. Sie zerschnitten oft sogar Beziehungen und politische Einheiten, haben sich jedoch fast unverändert über die Kolonialzeit hinaus erhalten - was, so die Autorin, mit dem Streben der kolonialen Eliten nach Machterhalt und den nach wie vor anhaltenden kolonialen Beziehungsmustern (zu den ehemaligen Mutterländern; wirtschaftlich, aber auch sprachlich bedingt) zusammenhängt.

Fronten im Sinne von Kampflinien, die sich aber auch weit in das Hinterland auswirken können, werden als eigener Typ genannt.

Phantomgrenzen sind dieser Typologie zufolge nicht mehr bestehende politische Grenzen, die nachwirken - wie beispielsweise der Karpatenbogen innerhalb Rumäniens als alte Grenze zwischen den ehemals habsburgischen und osmanischen Gebieten oder die Teilungsgrenzen Polens. Letztlich ist alles, was wir in der Geographie als Kulturlandschaften bezeichnen und keinen aktuellen politischen Einheiten entspricht, von Phantomgrenzen gesäumt: Erinnerungskulturen halten das Bewusstsein der alten Einheit und einer kulturellen Differenz aufrecht. In umgekehrtem Sinn wirkt in zerfallenen politischen Einheiten wie in der Sowjetunion, in Jugoslawien oder auch in der Habsburgermonarchie bei manchen Bevölkerungsgruppen (oft Minderheiten, die sich im neuen Nationalstaat nicht wohl fühlen) eine verklärte Erinnerung an den früheren Staat nach.

Kulturgrenzen bestehen der Typologie zufolge zwischen allen menschlichen Identitätsgruppen, worauf immer sich diese Identität beziehen mag. Jeder Mensch gehört auch mehreren Identitätsgruppen an, hat also multiple Identitäten. Sie wurden aber im Verlauf der Nations- und Staatenbildung immer mehr von nationalen Identitäten überlagert. Kulturelle Systemgrenzen gehen über Staaten und Nationen hinweg und beruhen zumeist auf Religionen und anderen Wertegemeinschaften. 
Sakralsprachen erleichterten im Fall durch Religion begründeter Systeme die staatenübergreifende Kommunikation. Europa teilt sich in dieser Hinsicht in eine lateinische und eine griechische Sphäre. Das lateinische Europa sehen wir, vielfach nicht bewusst, als das ,eigentliche` Europa an, es bildet auch den Kern der EU und letztlich des westlichen Gesellschaftssystems. Kulturelle Systemgrenzen gab es auch zur Zeit des in antagonistische Blöcke geteilten Europa. Nach dem Zerfall des Ostblocks haben sie sich nach Osten verschoben, wobei sich das westliche System heute als liberal-laizistisch-demokratisches mit einer Betonung individueller Freiheiten versteht.

Schließlich begegnen wir wirtschaftlichen und sozialen Grenzen, die vertikal (zwischen Menschen an einem Ort) und horizontal, räumlich (zwischen den Bewohnern verschiedener Orte) verlaufen können. Hier sind wir auf dem uns Geographen sehr vertrauten Feld der räumlichen Disparitäten bis hin zur Gentrifizierung und zu ,gated communities“.

Das Kapitel über Grenzregime und Politik der Grenze befasst sich zunächst ausführlich mit den Maut- und Zollsystemen, die die Warenströme regeln. Es zeigt deutlich, dass Zollprotektionismus auch die Kleinen leben lässt, während die Vergrößerung der Zollgebiete bis hin zum einheitlich regulierten, aber in Hinblick auf Produktivität doch so heterogenen Binnenmarkt der EU zu einem Überleben der wirtschaftlich Stärkeren führt und damit räumliche Disparitäten verstärkt.

Der Zusammenhang zwischen Zollpolitik und Wirtschaftsentwicklung wird auch an historischen Beispielen sehr deutlich. So konnte sich Frankreich unter Napoleon durch die Kontinentalsperre gegen die überlegene englische Industrie schützen und hatte selbst einen großen europäischen Binnenmarkt zur Verfügung. Heute setzen die großen Wirtschaftsmächte die Prinzipien von Freihandel (als Mittel der Erschließung von Märkten) und Protektionismus (um sich selbst zu schützen) wechselweise ein, um ihre Position gegenüber Schwellen- und Entwicklungsländern zu behaupten.

Was den Personenverkehr betrifft, rückt die Autorin zunächst die heute oft kolportierte Vorstellung zurecht, Migration wäre kein Ausnahmefall, sondern ebenso ,normal' wie Sesshaftigkeit und gewissermaßen das Öl der Weltwirtschaft. Sie meint, dass Migration im Gegenteil immer eines starken Auslösers (Push- oder/und Pullfaktors) bedürfe und viele Widerstände zu überwinden habe, in der früheren Geschichte vor allem auch den Raumwiderstand. Untertanen, später Staatsbürger, wären in der Geschichte in der Regel als Reichtum eines Staates betrachtet worden, auf den man nicht so gern verzichtete. Das ist ein Aspekt, der vielleicht auch in der heutigen Sichtweise auf Migration eine größere Rolle spielen sollte. Gleichwohl ist die Geschichte voll von freiwilligen und erzwungenen Migrationen, auch massenhaften Migrationsbewegungen. Pässe im heutigen Sinn gibt es erst seit der Herausbildung von Staaten heutigen Typs im 18. Jahrhundert. Ein ausführlicher Blick wird auf die unterschiedlichen Reiseregime im europäischen Westen und Osten von der Zeit nach dem Zweiten Weltkrieg bis zur politischen Wende 1989 geworfen. Sodann kommt die Problematik des Schengen-Regimes zur Sprache, das weitgehende Bewegungsfreiheit im Inneren, die aber auch Verlierer kennt, mit hohen Hürden nach außen („Festung Europa“) verbindet. Tatsächlich lassen sich die Verlierer innerhalb der Europäischen Union nach Aufhebung der Beschränkung der Arbeitskräftewanderung auch gegenüber Rumänien und Bulgarien im Jahr 2014 klar ausmachen: Es sind die Peripherien der EU mit niedrigen Löhnen, in denen - so wie eben in Rumänien und Bulgarien - infolge selektiver Abwanderung in die Zentren bereits Versorgungsengpässe im Gesundheits- und Bildungswesen sowie bei Facharbeitern auftreten, ein Faktum, das - wie auch die Autorin bemerkt - in der Diskussion um die europäische Integration kaum Beachtung findet.

Hochaktuell sind auch die Ausführungen über Asylrecht und Flüchtlinge im Zusammenhang mit der Migrations- und Flüchtlingswelle 2015, die sehr nüchtern und realistisch unter allen relevanten Aspekten geschildert wird - ein Abschnitt (S. 196-204), der auch für sich ungemein lesenswert ist. Sehr zu Recht weist die Autorin darauf hin, dass kaum jemand bei uns bedenkt, welche Lücke die Migranten und Flüchtlinge in ihrem Herkunftsgebiet reißen, dass sie der dortigen Gesellschaft 
fehlen und deren Potenzial zur Regeneration schwächen. Es ließe sich noch hinzufügen, dass dem verständlichen Streben nach persönlichem Glück wohl auch die Verantwortung für die eigene Gemeinschaft gegenübersteht - ein Gesichtspunkt, der besonders Kirchen und Caritas eigentlich sehr einsichtig sein müsste.

Grenzen des sozialen Auf- und Abstiegs bestimmen die vertikale soziale Durchlässigkeit einer Gesellschaft. Besonderes Augenmerk wird aber auf die räumliche Differenzierung sozialer Ungleichheit, auf sozio-ökonomische räumliche Disparitäten und deren Beständigkeit zwischen und innerhalb von Staaten gelegt.

Wer politische Grenzen überschreitet, quert immer auch kulturelle Grenzen. Diese bestehen aber natürlich auch innerhalb von politischen Einheiten, zwischen Subkulturen, z.B. Berufszweigen, die früher oft ethno-religiöse Merkmale aufwiesen. Sie setzen sich, so die Autorin, heute in kulturellen Netzwerken anderer Art fort, die für das berufliche Fortkommen wichtig und zum Teil international sind und zugleich inklusiv und exklusiv wirken. So wie früher manche Berufszweige kulturell geprägt waren, sind es heute Migrantengruppen im Zielgebiet, vor allem, weil sie Kommunikationsund Informationsnetzwerke bilden, die die Migration (an einen gemeinsamen Zielort) erleichtern. Im historischen Rückblick erweist sich auch deutlich die Beständigkeit von Migrationspfaden, die unter ähnlichen politischen und wirtschaftlichen Voraussetzungen immer wieder aufleben. Ebensolche Pfade existieren im Handel und bei Kapitalinvestitionen.

Im Schlusskapitel wird noch einmal auf ein zentrales Anliegen der Autorin hingewiesen, nämlich dem universalistischen eurozentrischen (man müsste eigentlich sagen: Westeuropa-zentrischen) Anspruch entgegenzutreten, der im Grunde genommen von allen bei uns heimischen Denkrichtungen, ob sie sich nun protektionistisch oder weltoffen geben, bewusst oder unbewusst vertreten wird. Dieses Anliegen zieht sich als Tenor durch das ganze Buch und gibt viel zu denken. Für ein gedeihliches Zusammenleben in dieser Welt werde es darauf ankommen, dem globalen Süden endlich zuzugestehen, vor allem auch im ökonomischen Bereich Grenzen zu setzen, die in seinem eigenen Interesse liegen.

Das sehr lesenswerte Buch ist global in der Perspektive, illustriert aber Vieles durch Beispiele aus Österreich und seiner Nachbarschaft. Diese lassen oft interessante historische Analogien zu aktuellen Situationen erkennen. Es ist ein großartiges Buch, trotz vieler Beispiele an thematischer Stringenz nicht zu überbieten und in seiner Beherrschung eines derart, grenzenlosen“ Themas bewundernswert; eine wichtige Horizonterweiterung für jeden Geographen, besonders aber für Kulturgeographen und Politische Geographen, durch eine Historikerin, die ,das Geographische` in sich hat, stark in räumlichen Bezügen denkt.

Was etwas überrascht, ist die geringe Beachtung, die Karten in Bezug auf das Festlegen von Grenzen und die Bewusstseinsbildung über Grenzen und Territorialität geschenkt wird. Haben nicht Karten, insbesondere ihre zivile Verwendung und Popularisierung ab dem späteren 19. Jahrhundert, vor allem auch durch Schul- und Handatlanten, die räumliche Vorstellung wesentlich verändert, die Wahrnehmungsräume erweitert und sehr zur Vermittlung der Selbst- und Fremdbilder von Staaten beigetragen?

Peter JORDAN (Wien)

Solarz Marcin Wojciech (Hrsg.) (2018): Geograficzno-polityczny atlas Polski. Polska w świecie wspólczesnym / Poland in the modern world. Atlas of Poland's political geography. Warszawa/Warsaw, Trzecia strona. 1. Aufl., 248 S., zahlr. farbige Karten. ISBN 978-83-64526-66-4.

Polen, einer der wenigen mittelgroßen Staaten der Europäischen Union (EU), wird in seiner geopolitischen Selbstsicht und Selbsteinschätzung nur selten gewürdigt. Daraus erwachsen Vorurteile und Animositäten. Einer der wesentlichen Aspekte dieser Selbstsicht ist die von der Gründung eines ersten 
polnischen Staates im 10. Jahrhundert an gegebene ,Sandwich-Position' zwischen einer starken Macht im Westen (zuerst dem Heiligen Römischen Reich, später Preußen und Deutschland) und einer starken Macht im Osten (zuerst dem Kiewer Reich, später dem Großfürstentum/Zarentum Moskau, Russland und der Sowjetunion, dann wieder Russland). Ein zweiter Aspekt ist das Trauma des Verschwindens von der Landkarte (1795-1918) nach jahrhundertelanger Staatlichkeit und der Aufteilung unter den Nachbarn Preußen, Österreich und Russland. Ein dritter ist die Aufteilung zwischen Hitler und Stalin im Zweiten Weltkrieg. Aus allen entstand ein tief wurzelndes Misstrauen gegenüber den heute noch (bzw. wieder) bedeutenden Mächten im Westen und Osten, das sich in aktuellen politischen Haltungen wie zum Beispiel in der Flüchtlingsfrage oder in der Gegnerschaft zur Ölpipeline ,North Stream‘ äußert, die Deutschland und Russland unter Umgehung Polens verbinden soll. Ein weiterer wichtiger Punkt in diesem Zusammenhang ist die starke Rolle der Katholischen Kirche, die wesentlich auf deren Funktion als ,Hort der Nation“ in der Zeit der Teilungen und auch als Sammelbecken der Dissidenz in der kommunistischen Periode zurückgeht. Sie lässt die Polen gegenüber manchen ,Segnungen ' der modernen westlichen Zivilisation skeptisch sein und erzeugt das Fremdbild eines übermäßig konservativen, im liberalen Westen noch nicht ganz , angekommenen“ Landes. Dem steht das Selbstbild eines fest in der westlichen Kultur verankerten Staates, ja eines Kernlandes des westlichen Gesellschaftssystems und des westlichen Christentums gegenüber, das sich gegen die Mongoleneinfälle, das byzantinisch geprägte Russland und das Osmanische Reich wiederholt als Bollwerk erwiesen hat. Polen fühlt sich außerdem als Mutterland einer weltweiten Diaspora, und die polnische Nation sieht sich damit als weit größer als es die Einwohnerzahl des Landes besagt.

Der vorliegende Atlas ist eine gute Gelegenheit, sich mit diesem polnischen Selbstbild näher auseinanderzusetzen. Denn Atlanten - insbesondere Schul- und Nationalatlanten, aber auch dieser aus Anlass von hundert Jahren seit dem Wiedererstehen eines unabhängigen Polen (1918-2018) und von 30 Jahren eines wieder freien Polen (1989-2019) herausgegebene und mit einem Vorwort des Staatspräsidenten Andrzej Duda versehene politisch-geographische Atlas - dienen (auch) der Selbstdarstellung eines Staates - gegenüber der eigenen Bevölkerung, aber auch, wenn sie wie dieser zudem in einer internationalen Verkehrssprache (Englisch) abgefasst sind, gegenüber einer internationalen Öffentlichkeit. Sehr treffend wird deshalb im einleitenden Teil auf den berühmten dreisprachigen (polnisch, deutsch, französisch) ersten Nationalatlas Polens von Eugeniusz Romer (1916) hingewiesen, der bei den Pariser Friedensverhandlungen wesentlich zum Wiedererstehen Polens beigetragen und eben diese Funktion der Repräsentanz eines Staates nach innen und außen beispielhaft erfüllt hat. Dies soll nicht heißen, dass solche Atlanten nicht nach wissenschaftlichen Grundsätzen erstellt und wissenschaftlich wertvoll sind. Aber schon die Auswahl der Themen, die kartographische ,Erzählung' der Geschichte des Landes und seines politischen Schicksals, die sich daraus ergibt, lenken die Gedanken und erzeugen ein gewolltes Bild.

Der Atlas ist zweisprachig polnisch-englisch in allen Texten, Kartentiteln und Kartenlegenden und damit über das Englische vollkommen erschließbar. Er wurde konzipiert und herausgegeben von M. W. Solarz, Professor für Geographie an der Universität Warschau [Warszawa], hatte aber auch viele weitere Mitarbeiter, die Karten entwarfen. Er gliedert sich in die Hauptkapitel „Internationale Beziehungen“, „der Staat“, „Gesellschaft" und „Entwicklung“.

Das Hauptkapitel „Internationale Beziehungen“ beginnt mit der Einbindung Polens in NATO und EU und mit der Frage nach der Eigenstaatlichkeit in verschiedenen Phasen der Geschichte. Unter den heutigen EU-Staaten gehört Polen zu jenen mit alter Eigenstaatlichkeit, nämlich seit der Wende vom 10. zum 11. Jahrhundert. Die territoriale Entwicklung Polens (vom Jahr 966 an) in Karten und mit einer historisch kommentierten Zeitachse zeigt die historische Hauptexpansionsrichtung nach Osten, die Westverschiebung nach dem Zweiten Weltkrieg und den häufigen Herrschaftswechsel auf dem Territorium des heutigen Polen. 
Wie immer sind Karten, die die historische Dauer von internationalen Grenzen zeigen, besonders eindrucksvoll und aussagekräftig. Dies ist auch bei Polen so, das von einer 203 bis 226 Jahre währenden Grenze durchschnitten wird, nämlich der Grenze zu den ehemals deutschen Ostgebieten. Sie steht der längstwährenden heutigen Außengrenze (jener entlang der Ostseeküste östlich von Danzig [Gdańsk]) nur um 71 Jahre nach.

Geradezu frappierend ist, wie sehr sich die Teilungsgebiete bis heute in Bezug auf viele demographische, soziale und wirtschaftliche Indikatoren unterscheiden. Das wird besonders eindrucksvoll auch durch das höchst unterschiedliche Wahlverhalten (gezeigt anhand der Parlamentswahlen 2005, 2007, 2011, 2015) diesseits und jenseits einstiger Teilungsgrenzen belegt. Man kann dies mit der außerordentlich formativen Wirkung des 19. Jahrhunderts und der Zeit bis zum Ersten Weltkrieg durch Eisenbahnbau, Industrialisierung und Urbanisierung erklären, während derer Polen zwischen Staaten sehr unterschiedlichen Entwicklungsniveaus aufgeteilt war.

Als für die polnische Außenpolitik und Geschichte maßgebliche geographische Faktoren werden die Lausitz-Stettiner Pforte entlang der Neisse und unteren Oder gegenüber dem heutigen Deutschland, die Gebirgszüge der böhmischen Randgebirge und der Karpaten, die Pforte von Przemyśl zur heutigen Ukraine, der Isthmus zwischen Ostsee und Schwarzem Meer und die Ostsee genannt.

Auf einer einseitigen Serie kleiner Karten wird die Funktion Polens als ein Bollwerk des politischen und gesellschaftlichen Westens von den Mongoleneinfällen im 13. Jahrhundert bis in die Zwischenkriegszeit und zum Zweiten Weltkrieg (1920 gegen Sowjetrussland, 1939 gegen die Sowjetunion und Nazi-Deutschland, 1944 im Warschauer Aufstand gegen die Sowjetunion) und in die Gegenwart (seit 1999 bzw. 2004 östlicher Vorposten von NATO und EU gegenüber Russland mit dessen Ansprüchen auf Sonderrechte im „Nahen Ausland“) betont.

Sehr außergewöhnlich sind zwei Karten von Lehnwörtern aus anderen Sprachen im Polnischen und von polnischen Lehnwörtern in anderen Sprachen als Ausdruck internationaler Beziehungen. So stammen von den 26,2 Prozent Wörtern fremden Ursprungs in der heutigen polnischen Standardsprache 36,6 Prozent aus dem Lateinischen, 19,7 Prozent aus dem Deutschen, 15,9 Prozent aus dem Französischen und 7,1 Prozent aus dem Tschechischen. Man erkennt daran die Vorbildwirkung der prestigebehafteten westlichen Verkehrs- und Nachbarsprachen. Latein war außerdem bis zu den polnischen Teilungen im späten 18. Jahrhundert die nominelle Amtssprache Polens. Die polnische Sprache ihrerseits beeinflusste am meisten das Russische und Tschechische, nur noch mäßig das Litauische und Rumänische und kaum noch das Ungarische, Slowakische und Deutsche (z.B. Grenze, Kummet, Jauche, Peitsche, Gurke). Die Prägekraft des Lateinischen wird auch in den polnischen Exonymen für Ländernamen deutlich, die sehr oft direkt aus dem Lateinischen übernommen wurden - wie Austria für Österreich.

In der ebenfalls sehr seltenen Karte des sprachlichen Ursprungs von Flussnamen zeigt sich das für Europa typische Bild vieler vorslawischer, keltischer Namen, besonders für die größeren Flüsse.

Diagrammhaft dargestellt ist die Stellung Polens als Land des Übergangs von West nach Ost nach heutigem Lebensstandard und heutiger Wirtschaftskraft. Es rangiert immer deutlich hinter Deutschland und oft auch noch hinter Tschechien, aber immer vor Weißrussland, der Ukraine und Russland.

Sehr aufschlussreich sind Karten der EU nach den Ergebnissen einer Gallup-Umfrage 20142016, die die Befürwortung oder Ablehnung einer Weltführerschaft verschiedener Mächte erhob. Nur Deutsche und Polen sprachen sich mehrheitlich für eine Weltführerschaft der EU aus; alle anderen EU-Staaten außer Italien, Spanien, Portugal, Griechenland und Zypern für eine Deutschlands; Italien, Spanien und Portugal für eine der USA; und Griechenland und Zypern für eine Russlands.

Die slawischen Länder, die Ostseeanrainer, die Visegrad-4-Staaten plus Rumänien, die Nachfolgestaaten der „Königlichen Republik“ „Polen-Litauen“ (Polen, Litauen, Weißrussland, Ukraine) werden als Ländergruppen nach aktuellen Daten vergleichend thematisch behandelt. „Polen-Litau- 
en“ hätte demzufolge im Jahr 2015 mehr Einwohner als Deutschland (98 gegenüber 81 Millionen). Man kann sich fragen, ob eine ähnliche Zusammenfassung der Nachfolgestaaten der Habsburgermonarchie in einem österreichischen Atlas denkbar ist? Die Gebiete des einstigen „Polen-Litauen“ sieht Polen auch heute wieder als sein engeres politisches Aktionsfeld an, was auch in der Häufigkeit von Staatsbesuchen zum Ausdruck kommt.

Unter dem Titel „Polish soft power“ wird eine Weltkarte mit geographischen Namen, die sich auf Polen beziehen, geboten. Sie sind natürlich in den Zielgebieten polnischer Auswanderung (USA, Kanada, auch Südamerika und Australien) besonders zahlreich, wohin polnische Auswanderer ,ihre“ Namen mitgenommen haben.

Unter den Karten zur Religion weist eine Karte der Atheisten und Agnostiker Polen als das EULand mit der größten Religiosität neben Irland und Rumänien aus.

Im Hauptkapitel über den Staat wird zunächst das politische System Polens im Vergleich mit den anderen EU-Staaten geschildert. Außergewöhnlich ist eine Karte der ehrenamtlichen Tätigkeiten auf der Grundlage einer Gallup-Umfrage in den Jahren 2014-2015. Ihr zufolge liegt Polen sogar unter dem Niveau der meisten Transformationsländer, die (mit Ausnahme Sloweniens) ohnehin gegenüber Mittel- und Westeuropa weit hinten liegen. In Bezug auf den „Gender pay gap“ (2015) zeigt sich hingegen ein großer Vorsprung Polens (5-7,5\%) gerade gegenüber Österreich, das wie Deutschland, Tschechien, Estland, das Vereinigte Königreich und Portugal mit 25,1-30,0 Prozent einen Spitzenwert in der EU aufweist.

Karten zur Gliederung des Landes in Verwaltungsgebiete dokumentieren den häufigen Wechsel seit 1945, zuletzt mit der Einführung der größeren und historisch-kulturellen Identitäten gut entsprechenden Woiwodschaften im Jahr 1999. Sehr ausführlich werden Wahlergebnisse referiert. Es erweisen sich große Unterschiede zwischen den ehemaligen Teilungsgebieten, wobei im einst russischen und österreichischen (Galizien) Teilungsgebiet strukturkonservativer gewählt wird. Wie in den meisten Transformationsländern leidet auch Polen unter geringer Wahlbeteiligung (unter 50\% bei nationalen Wahlen, unter 30\% bei Europawahlen).

Gedenknamen nach Kommunisten und kommunistischen Institutionen und Ereignissen im öffentlichen Raum (v. a. für urbane Verkehrsflächen) können als ein Ausdruck kommunistischer Nostalgie interpretiert werden und zeigen eine Häufung im oberschlesischen Industriegebiet. Gedenknamen nach Personen, Institutionen und Ereignissen des unabhängigen Polen (der Zwischenkriegszeit und der Zeit nach der politischen Wende) sind weit verbreitet und zeigen keine auffallende regionale Häufung. Papst Johannes Paul II. (Karol Wojtyła), Kardinal Stefan Wyszyński, Władysław Sikorski, Militär und Politiker vom Ersten bis in den Zweiten Weltkrieg und Vorkämpfer für die polnische Unabhängigkeit, sowie Józef Piłsudksi, autoritär regierender Staatschef von 1926 bis 1935, sind die Personen, nach denen am häufigsten benannt wurde. Es erweist sich die starke Stellung der Katholischen Kirche.

Das Hauptkapitel zur Gesellschaft beginnt mit demographischen Karten und wendet sich dann den polnischen Staatsbürgern im EU-Ausland im Jahr 2015 zu: Vereinigtes Königreich 870.000, Deutschland 640.000, Irland 110.000, Niederlande 100.000, Italien 90.000, Norwegen 85.000, Spanien 65.000, Belgien 65.000, Österreich 55.000, Schweden 50.000. Gebürtige Polen im EU-Ausland sind natürlich noch zahlreicher.

Der Anteil der im eigenen Land Geborenen in den EU-Ländern im Jahr 2015 zeigt Österreich mit Schweden und Irland in der niedrigsten Stufe (55-80\%), Polen mit fast allen anderen Transformationsländern (außer Kroatien, Estland, Lettland) in der höchsten (95-99\%). Das sind die Auswanderungsländer nach 1989. Die geringe Vertrautheit mit Zuwanderung und Integration erklärt auch teilweise die Weigerung dieser Länder, sich 2015 an der Aufteilung der Flüchtlinge und Migranten 
$\mathrm{zu}$ beteiligen. Es folgen weitere Karten zur Migration, darunter eine Typisierung der Migration in den EU-Ländern.

Sehr ausführlich werden die nationalen und ethnischen Minderheiten in Polen, deren Verteilung und demographische Entwicklung abgehandelt. Die zahlenmäßige Zu- und Abnahme der Minderheiten spiegelt kaum die natürliche Bevölkerungsbewegung, sondern vor allem das Bekenntnis, die subjektive Zuordnung, wider. Es nimmt bei den Kaschuben zu, bei den Deutschen und Weißrussen ab. Karten zu geographischen Namen in Minderheitensprachen im öffentlichen Raum und ein Vergleich zwischen nationalem/ethnischem Bekenntnis und Stimmabgabe für Minderheitenparteien ergänzen diesen Abschnitt.

Karten zu Polen in der Welt auf der Grundlage von Schätzungen des Vereins „Polnische Gemeinschaft" schließen auch Personen polnischer Abstammung nach vielen Generationen mit ein, entsprechen also einem sehr weiten Nationsbegriff, der die subjektive Zuordnung außer Acht lässt. So ergeben sich zirka 10 Millionen Polen allein in den USA und wohl insgesamt 20 Millionen in der ganzen Welt, was bedeutet, dass die polnische Nation ,in Wirklichkeit“ 60 Millionen umfasst und nur zwei Drittel von ihr im Mutterland leben. Eine Karte von Polen in Europa beruht auf der gleichen Grundlage und zeigt, dass - in absoluten Zahlen - in Deutschland und Frankreich die meisten Polen leben, gefolgt vom Vereinigten Königreich, Weißrussland und der Ukraine. Die größten ,„polnischen Städte“ außerhalb Polens sind nach demselben Prinzip 1. New York (207.000 Polen), 2. Chicago (160.700), 3. London (136.000), 4. Berlin (101.100), 5. Toronto (98.300), 6. Wilna [Vilnius] (85.800), 7. Hamburg (71.300), 8. Grodno [Grodna] (64.600), 9. Wien (51.600), 10. Winnipeg (50.400).

Auch den autochthonen polnischen Minderheiten in anderen Ländern wird mit detaillierten Verteilungskarten viel Raum gewidmet. Sie befinden sich in Litauen, Weißrussland, Lettland, Ukraine, Tschechien (Teschener Gebiet) und Rumänien (Bukowina [Bucovina]). Bei den Polen in Deutschland (Ruhrgebiet!), im Vereinigten Königreich, in den USA, Kanada und Kasachstan kann man vielleicht doch nicht von autochthonen Minderheiten sprechen.

Keine Beachtung finden dagegen - anders als im 1997 in Breslau [Wrocław] erschienenen Regionalatlas von Nieder- und Oppelner Schlesien [Atlas Śląska dolnego i opolskiego] - der Bevölkerungsaustausch in den ehemals deutschen Ostgebieten und auch die drastische Reduktion der jüdischen Bevölkerung im Holocaust, die einst ein prägendes Element polnischer Stadtkultur war.

Das abschließende Hauptkapitel zur Entwicklung widmet sich zunächst dem Human Development Index (HDI) und zeigt Veränderungen des HDI 1990-2015 für die Staaten der EU. Polen steht diesbezüglich nach Kroatien an der Spitze der Transformationsländer. In der Summe der Wohlstandsindikatoren erweist sich Polen ebenso wie die anderen Transformationsländer aber als immer noch schwach im Vergleich zum westlichen Europa. Auch bei den Umweltindikatoren liegt Polen (mit den meisten Transformationsländern) immer noch an der Spitze der Emittenten unter den EU-Ländern. Unter den Wirtschaftsindikatoren fällt vor allem die Produktivität auf. Sie ist in allen Transformationsländern im Vergleich zur Rest-EU (selbst zu Italien, Spanien und Griechenland) immer noch (2016) sehr niedrig, Polen liegt im Mittelfeld der Transformationsländer.

Ein zweisprachiges (polnisch-englisches) Ortsnamenverzeichnis und ein ebensolches Verzeichnis der Namen politischer Parteien runden den Atlas ab. Er kann als außergewöhnlich und aus den heutigen thematischen Atlanten hervorstechend bezeichnet werden. Sein Themenkanon und auch manche seiner kartographischen Lösungen werden sicher bespielgebend wirken. Unter den für politisch-geographische Atlanten ungewöhnlichen und bemerkenswerten Themen finden sich viele toponomastische und linguistischen Karten, die wohl auf die Mitwirkung von Maciej ZYch, Toponomast und Vertreter Polens in der Expertengruppe der Vereinten Nationen für geographische Namen (United Nations Group of Experts on Geographical Names, UNGEGN), bzw. der Linguistin Urszula 
BIJAK zurückzuführen sind. Die Vielzahl an Karten, die Polen als Teil der EU und im Vergleich mit anderen EU-Ländern zeigen, kann man als Bekenntnis zu Europa und zur EU deuten. Kartographisch-methodisch ist der Atlas modern, gut lesbar und einprägsam gestaltet. Lediglich die farbigen Raster der EU-Karten könnten sich manchmal etwas deutlicher unterscheiden.

Peter JORDAN (Wien)

Weichhart Peter, Rumpolt Peter A. (Hrsg.) (2015): Mobil und doppelt sesshaft. Studien zur residenziellen Multilokalität (= Abhandlungen zur Geographie und Regionalforschung, Band 18). Wien, Institut für Geographie und Regionalforschung der Universität Wien. 1. Aufl., 344 S., zahlreiche z. T. farbige Abb. und Tab. ISBN: 978-3-900830-85-4.

Residenzielle Multilokalität, verstanden als spezifische sozial-räumliche Alltagspraxis von Einzelpersonen, Familien oder Haushalten über zwei oder mehrere Wohnstandorte hinweg, ist historisch gesehen nichts Neues. War sie lange ein Phänomen, das die Lebensweise von Eliten, sozial Benachteiligten oder bestimmten Berufsgruppen kennzeichnete, so ist das Wohnen oder Leben an mehreren Orten im Zuge des gesellschaftlichen Wandels in der Spätmoderne zu einem Phänomen geworden, das von zahlreichen Menschen in unterschiedlichen Lebensphasen ausgeübt wird - wenn auch oft nur für eine begrenzte Zeit. Mit dieser Entwicklung ist das multilokale Wohnen seit einigen Jahren zunehmend in den Fokus der Forschung gerückt (Wood et al. 2015).

Der vorliegende Sammelband vereint elf Beiträge, in denen verschiedene Konzepte, theoretische und methodische Überlegungen sowie empirische Befunde zu diesem relativ jungen Themenfeld vorgestellt werden. Fast alle der 16 Autorinnen und Autoren engagieren sich seit Jahren im internationalen „Netzwerk Multilokalität“ (https://www.uni-muenster.de/ Geographie/Multilokalitaet/home.html), das sich in den Jahren 2006/07 formiert hat. Sie vertreten unterschiedliche sozial-, kultur- und raumwissenschaftliche Disziplinen.

Die Herausgeber Peter WeichHart und Peter A. Rumpolt streben mit dem Band „bewusst eine Art Collage“" (S. 8) und keine systematische Darstellung an, um der Vielfalt der Ausprägungsformen des Phänomens der residenziellen Multilokalität gerecht zu werden sowie die Breite der möglichen Zugangsweisen und Forschungsfragen sichtbar zu machen. Das ist ihnen mit dem vorliegenden Buch auch gut gelungen. In ihrem sehr lesenswerten einführenden Artikel thematisieren sie die allgemeine Relevanz des Forschungsfeldes, terminologische Herausforderungen sowie Probleme der Datenverfügbarkeit. Vertiefend setzen sie sich mit den im Kontext der residenziellen Multilokalität sehr bedeutsamen Konzepten des „Wohnens“ sowie der Ortsbindung und der „Place Identity“ auseinander, zeigen unterschiedliche Ansätze der Typisierung von Ausprägungsformen residenzieller Multilokalität auf und geben eine Übersicht über mögliche theoretische Anknüpfungspunkte. In dem Beitrag werden zahlreiche Desiderata der Forschung angesprochen, u. a. die Rückwirkungen, die multilokale Wohnformen auf sozioökonomische und raumstrukturelle Gegebenheiten haben. Im zweiten Beitrag vertieft Peter WeIchHART bereits im ersten Artikel aufgeworfene terminologische Fragen und thematisiert Probleme der Abgrenzung von residenzieller Multilokalität zu ähnlichen Konzepten und Begriffen (wie Translokalität, Nomadismus, zirkulärer Migration, Migration und Zirkulation). Er geht außerdem auf die Bedeutung der Konzepte von An- und Abwesenheit, passiven und aktiven Akteuren sowie die Bedeutung eines Raumes „Dazwischen“, des „Transitionsraums“, als wichtige Aspekte von residenzieller Multilokalität ein.

Die folgenden vier Beiträge widmen sich auf der Basis von quantitativen Methoden der Verbreitung und Beschreibung residenzieller Multilokalität. Alexander WiSBAUER et al. sowie Andrea DitTrich-Wesbuer et al. beschäftigen sich mit den Möglichkeiten, Herausforderungen und Gren- 
zen, die amtliche Statistiken und andere verfügbare Datenquellen zur Erforschung residenzieller Multilokalität in Österreich und Deutschland bieten. Während Caroline KRAMER empirische Befunde zum multilokalen Leben von Studierenden und Beschäftigten des Karlsruher Instituts für Technologie präsentiert, fokussieren Helmut SCHAD et al. auf das Aufkommen und die Ausprägungen multilokalen Wohnens in der Schweiz. In beiden Beiträgen wird deutlich, wie aufschlussreich es ist, wenn Daten zur Nutzung, Alltagsgestaltung und Aneignung aller Wohnstandorte erhoben und analysiert werden.

Im Zentrum der nächsten fünf Beiträge stehen schließlich neuerlich konzeptionelle Fragen. Sarah KRомPноLтZ verweist auf die nur eingeschränkte Passung des Begriffs des „Haushalts“ zur Untersuchung von residenzieller Multilokalität und schlägt ergänzend den Begriff der „Residenzgemeinschaft“" vor (S. 211). Cédric DuchÊNE-LACroix setzt sich mit Raumpraktiken, Ortsbezügen und Territorialitäten multilokal Lebender auseinander und schlägt dafür die Nutzung des Konzepts des „Archipels“ vor (S. 224). Knut Petzold bringt mit seinem Beitrag „Konzepte der soziologischen Ungleichheitsforschung in die aktuelle Debatte zur Multilokalität" ein (S. 240). Mit einer systematisierenden Darstellung gelingt es ihm, diese für die Multilokalitätsforschung fruchtbar zu machen. Helmut SCHAD, der leider im Jahr 2017 verstorben ist, präsentiert in seinem umfassenden Beitrag überzeugend, wie materielle Aspekte multilokalen Wohnens mit Bezug auf die Akteursnetzwerktheorie konzeptionell und methodisch fruchtbar in den Blick genommen werden können. Im letzten Beitrag des Sammelbandes thematisiert Nicola Hıtr schließlich aus sozialwissenschaftlicher Perspektive Gedanken zu wohnungswirtschaftlichen Implikationen unterschiedlicher Formen multilokalen Wohnens und verweist damit auf vielfältige weitere offene Forschungsfelder der Multilokalitätsforschung.

Der Sammelband bietet insgesamt einen guten Einblick in den Stand der terminologischen und konzeptionellen Diskussionen im Forschungsfeld „residenzielle Multilokalität“ im deutschsprachigen Raum. Liest man die Beiträge nacheinander, bemerkt man allerdings so manche Redundanzen und Überschneidungen, vor allem im Bemühen der Abgrenzung und Beschreibung des Untersuchungsgegenstands. Besonders positiv hervorzuheben ist, dass der Band gerade durch die interdisziplinäre Herangehensweise das breite Spektrum von Fragestellungen sichtbar macht und sehr unterschiedliche spannende empirische Befunde präsentiert. Er zeigt schließlich zahlreiche offene terminologische, konzeptionelle und empirische Fragen und Forschungsbedarfe auf, an die Leserinnen und Leser in ihren eigenen Forschungen anknüpfen können.

\section{Zitierte Literatur:}

Wood G., Hilti N., Kramer C., Schier M. (2015): A Residential Perspective on Multilocality. Editorial. In: Tijdschrift voor economische en sociale geografie (TESG), 106, 4, S. 363-377. 